conditions that are so confining as to distort the phenomena studied and to miss their critical aspects.

A restrictive approach can actually subvert symbolic processes; for example, it is impossible to study representational ability in apes without providing a rich ecological, ethological, and symbolic context of enculturation (Miles 1997; Savage-Rumbaugh \& Lewin 1994). Researchers may need to act "as if" as a methodological requirement in order to determine whether animals have mental abilities. In fact, rich symbolic and social contexts and rewards may be crucial for understanding human biosocial and mental development (Shore 1996), as well as being a critical component of a normal rearing history for all hominoids. Social rewards in closely interactive units are certainly the norm in the development of socially competent chimpanzees (Goodall 1986).

Most important, to argue that only one method can allow us to conclude that children or animals do or do not have a theory of mind leads to other surprisingly narrow-minded analyses. For example, Heyes questions that the home raised chimpanzee, Viki, could imitate (Hayes \& Hayes 1952) on the grounds that no method was reported in which the experimenter measured the degree of similarity of Viki's behavior. But Heyes fails to note that this was later provided by a replication of this study with the enculturated orangutan Chantek (Miles et al. 1996). Heyes also considers only mirror self-recognition, and ignores other measures of self-awareness, including personal pronouns and possession (Itakura 1994; Miles 1994; Patterson \& Cohn 1994) as well as Menzel's (1974) experiment on chimpanzee deception. Finally, she ignores that all child studies are linguistically mediated either by virtue of the procedures used or by the prior enculturation of the children. Such adherence to a single perspective may hold some benefits, but overall it is myopic.

\section{Primate theory of mind is a Turing test}

\section{Robert W. Mitchella and James R. Andersonb}

aDepartment of Psychology, Eastern Kentucky University, Richmond, KY 40475. psymitch@acs.eku.edu; bDepartment of Psychology, University of Stirling, Stirling FK9 4LA, Scotland. j.r.anderson@stir.ac.uk

Abstract: Heyes's literature review of deception, imitation, and selfrecognition is inadequate, misleading, and erroneous. The anaesthetic artifact hypothesis of self-recognition is unsupported by the data she herself examines. Her proposed experiment is tantalizing, indicating that theory of mind is simply a Turing test.

We agree with several of Heyes's ideas, especially those which remind us of earlier arguments that self-recognition and bodily imitation need not imply theory of mind (Mitchell 1993), and that information about an animal's history is necessary to interpret its deceptions (Mitchell 1986; Morgan 1894). Our own research, replicating Woodruff \& Premack's (1979) chimpanzee study with capuchin monkeys, supports the idea that communicative and deceptive pointing can derive from discrimination learning (Mitchell \& Anderson 1997), though it raises the question of why macaques failed to learn deceptive pointing in a similar experimental setting (Blaschke \& Ettlinger 1987). Perhaps a more accurate and thorough literature review than the one provided by Heyes would establish more common ground.

Heyes claims that all instances of imitation in nonhuman primates "could" or "may" have come about by chance or nonimitative means, even when the same animal produces a variety of responses supporting a generalized ability for the imitation of behavior. Instead of evidence, scenarios of possible or spurious reinforcements for each particular instance are offered. Such speculations are not evidence against an ability for imitation; even in humans, imitation develops from nonimitative processes and generalization of previously learned behaviors (Guillaume 1926/1971). Alternative explanations for single behaviors produced by rats and budgerigars are ignored, no evidence is pro- vided that they can imitate a variety of actions, and their imitations appear unrelated to cross-modal imitations present in humans and some apes (Mitchell 1996; 1997). Relevant studies on primate imitation are ignored by Heyes: one experiment explicitly designed to shape imitative responses resulted in failure with a macaque (Mitchell \& Anderson 1993), another in success with an orangutan (Miles et al. 1996). Contrary to Heyes's claims, Gallup (1982) and Povinelli (1987) never discuss imitation as evidence of theory of mind, and only two (not all four) chimpanzees in Woodruff \& Premack's (1979) study pointed to an empty container with the competitor, and those on less than $80 \%$ of the last 60-94 trials, after about 190 trials each with competitor and cooperator.

In presenting the self-recognition literature, Heyes ignores evidence from apes of self-exploration of body-parts not visible without a mirror, studies using sham marking instead of anaesthesia, evidence of self-recognition in gorillas, and methodological flaws in studies of human infants (Parker et al. 1994). Contrary to her claims, studies using variants of the traditional mark test procedure do not support her anaesthesia artifact hypothesis, as these studies used monkeys (which never pass any form of the mark test) or very young great apes (see Gallup et al. 1995). Given that Heyes's hypothesis is intended to explain why chimpanzees pass the mark test, her inclusion of mark-directed touches by chimpanzees failing the mark test (in the means and standard deviations for the bimodal distribution created by combining both passing and failing animals) is baffling. For Swartz and Evans (1991) the one chimpanzee who passed the mark test (on all 3 trials) showed from 10-23 mark touches while looking at herself in the mirror, and from 1-7 mark touches in the control condition; for Povinelli et al. (1993), the 10 chimpanzees who passed the mark test touched the mark on average 11.2 times $(\mathrm{s}=10.8)$ in the mirror condition, but only 1.6 times $(\mathrm{s}=2.5)$ in the control condition (and including "spurious" mark-rubs does not change the difference between conditions). By contrast, those chimpanzees who failed the mark test showed infrequent mark touching, but more of it in the control condition than in the mirror condition, indicating that chimpanzees are not, generically, more likely to touch the mark by chance in the mirror condition than in the control. For Swartz and Evans, the 10 "failing" chimpanzees touched the mark infrequently in the mirror condition (21 tests with no touches, 5 with 1-4 touches), whereas most touched the mark in the control condition (11 tests with no touches, 15 with 112); for Povinelli et al., 18/20 of these chimpanzees failed to touch the mark at all in the mirror condition, but 12/20 touched it at least once (range 1-13) in the control condition (and including "spurious" mark-rubs just increases the latter range). For most chimpanzees, then, mark-touching frequency actually decreased as the time interval since anaesthesia increased.

Heyes argues that to detect itself via a mirror, an organism must distinguish its own bodily inputs from external inputs. The immediate relevance of this internal/external distinction to selfrecognition remains unclear (Anderson \& Gallup, in press; Mitchell 1996): human children make this distinction by 5 months of age (Watson 1994), yet do not show any signs of self-recognition until 10-19 months later. The logical conclusion from Heyes's argument is that all visually capable organisms should pass the mark test, yet they do not. Although she cites Epstein et al.'s (1981) "self-recognizing" pigeon experiment favorably, it has failed to replicate despite extensive efforts (Thompson \& Contie 1994). Heyes's assertion that autistic children show self-recognition at the same age as normal children is surprising, given that the youngest autistic children tested are 3-year-olds (Mitchell 1997).

Heyes's proposed experiments raise some surprising issues about theory of mind and about how a chimpanzee might interpret human actions. They presume that laboratory chimpanzees believe that humans know where objects are located solely through vision; yet these chimpanzees presumably have numerous experiences in which humans appear to know the location of something without having seen it. Indeed, in the second (variant) test, the human who does not observe the baiting or wears opaque goggles 
nevertheless knows enough to point to a container, and sometimes even to the correct one, which seems confusing. Greater frequency of correct solutions with Knower than with Guesser, or faster learning with Group Direct than with Group Reverse, do not seem adequate as evidence of theory of mind unless the correct solutions occurred from the start of the transfer trials. In fact, surely only a correct choice on the first transfer trial can count toward evidence of theory of mind, whether or not chimpanzees are consistently rewarded: reward would contaminate subsequent responses, and lack of reward could indicate error and therefore lead the chimpanzee to respond to other stimuli. Either way, simple task analysis indicates that the experiment she describes remains essentially a multiple discrimination learning task, which even macaques can perform (see Mitchell \& Anderson 1997). Perhaps, in fact, we can never be sure whether an animal is responding to another based only on the other's behavior, or on mental-state inferences from that behavior. As Turing (1950, p. 446) argued, we may have to maintain the "polite convention that everyone thinks" until his or her behavior suggests otherwise.

ACKNOWLEDGMENT

We thank Karyl Swartz for her assistance.

\section{Primate cognitive neuroscience: What are the useful questions?}

\section{A. Parker \\ Department of Experimental Psychology, University of Oxford, OX1 3UD, United Kingdom. amanda.parker@psy.ox.ac.uk}

Abstract: Study of "theory of mind" in nonhuman primates is hampered both by the lack of rigorous methodology that Heyes stresses and by our lack of knowledge of the cognitive neuroscience of nonhuman primate conceptual structure. Recent advances in this field indicate that progress can be made by first asking simpler research questions.

In the two decades that have elapsed since Premack and Woodruff (1978) opened the debate about theory of mind in nonhuman primates (NHPs), a great deal has been learned about the development of social intelligence in human infants. Heyes attributes the contrasting lack of progress in our understanding of NHP theory of mind to ineffectual experimental methodologies and lack of clear theorizing. To some extent she may be right. But another interpretation of the research covered in her target article might be that, in the present state of knowledge about NHPs, we are asking the wrong questions, or trying to force comparisons that are simply inappropriate. This is particularly true as theories of human theory of mind development have become sophisticated very quickly.

One recent proposal is that theory of mind development in human infants depends on the development of "modules" for intentionality detection, gaze direction detection, and shared attention, leading to the development of a further theory of mind module (Baron-Cohen 1995). The importance of linguistic processing in this last stage should not be underestimated. A useful research question would therefore be, To what extent do these modules, or their precursors, occur in NHPs? Heyes's proposed experiment deals with one aspect of visual attention, the inference of another's knowledge of an event from a calculation of whether or not they have perceived it. Her proposed method is likely to produce valuable insights on this topic. At the present point, we need precise quantitative knowledge about the range of NHP conceptual abilities about other minds.

Abilities are likely to be tied to neuroanatomy. So we should also ask about the extent to which different species of primates have similar or different neural structures and connections, and to what extent this leads to similar abilities. Neuroanatomical study of the frontal lobes of humans and macaque monkeys has revealed that their basic architectonic plan is the same (Petrides \& Pandya
1994). A recent comparison of cytoarchetectonic areas thought to be uniquely human with chimpanzee and macaque brains, likewise suggests that the basic organization of these areas is the same (Passingham 1997). Moving from anatomy to behavior, we have found that damage to structures known to be important in episodic memory in humans causes large deficits in object-in-place memory in monkeys (Parker \& Gaffan 1997a; 1997b). Similarly, damage to perirhinal cortex will affect the monkey equivalent of semantic memory - conceptual knowledge about objects. (Parker \& Gaffan 1997c). It is unlikely however, that autobiographical memory will develop without language (Nelson 1993), and it seems likely that autobiographical, rather than episodic, memory is the key feature of the human conception of the self and the consequent full development of complex representations of other minds.

Primates are able to understand the world, including their social world, because of their highly developed conceptual resources. An emergent property of the neural structure of the temporal and frontal lobes is that they store information as categories. In the temporal lobe, these structures represent objects, while the frontal lobe stores action, intention, and affect related schemata. Combining these two types of information enables primates to produce complex and subtle behaviors over a wide range of situations. At a certain point this conceptual structure may become elaborate enough to sustain a theory of mind. What are its basic building blocks? This is a more answerable question. One source of answers may lie in the way that object representations in the temporal lobe become integrated with appropriate strategies in the frontal lobe, and the effects of damage to these structures on this knowledge. Current experiments involve only abstract stimuli and arbitrary categories (Parker \& Gaffan 1997d) and indicate that when interaction between object knowledge and action strategies is prevented, behavior is at chance levels. Future research with this paradigm will examine real categories of objects. It is a short step from here to the study of how monkeys apply strategies to categories of conspecifics.

To conclude, much of the available evidence points to language as being vitally important for the development of a complete human theory of mind. This does not mean that components of this ability are not present in NHPs, but it does mean that using anthropomorphic methods to search for them is inappropriate. We should be searching for the basic elements of social understanding in primates, and using rigorous experimental methodologies, as Heyes suggests.

\section{To see or not to see, that is the question: Designing experiments to test perspective- taking in nonhumans}

Irene M. Pepperberg

Department of Ecology and Evolutionary Biology and Department of Psychology, University of Arizona, Tucson, AZ 85721.

imp@biosci.arizona.edu

Abstract: Heyes argues that we need alternative experiments to study those animal abilities generally considered to involve "theory of mind." The studies she proposes, however, have as many problems as those that she criticizes. Further interactions should exist among researchers examining these capacities before additional experiments are undertaken.

As a researcher who studies cognitive and communicative capacities of nonhumans (e.g., Pepperberg 1990; 1996), I both agree and disagree with Heyes's target article. I agree that questions concerning animal capacities such as self-awareness and perspectivetaking are intriguing, but that much published research into these areas has either failed to show such abilities or made unfounded claims for these capacities because of problems in experimental design. I disagree with some specific criticisms that Heyes has offered; because many of my colleagues have previously engaged 